Conclusions Our cohort showed high rate of patient satisfaction with telemedicine healthcare. However, the relatively low healthcare provider satisfaction rate raises concern as to whether telemedicine constitutes a satisfactory alternative to conventional in-person care. Additional researches are required to investigate the feasibility of telemedicine in long-term disease activity evaluation and patient outcome measurement.

\section{PREDICTING ADVERSE PREGNANCY OUTCOMES IN WOMEN WITH SYSTEMIC LUPUS ERYTHEMATOSUS: A COMPARISON OF MACHINE LEARNING METHODS}

${ }^{1}$ Melissa Fazzari, ${ }^{2}$ Marta Guerra, ${ }^{2,3}$ Jane Salmon, ${ }^{1}$ Mimi Kim*. 'Albert Einstein College of Medicine, New York, USA; ${ }^{2}$ Hospital for Special Surgery, New York, USA; ${ }^{3}$ Weill Cornell Medical College, New York, USA

\subsection{6/lupus-2021-lupus21century.49}

Background Nearly 20\% of pregnancies in patients with Systemic lupus erythematosus (SLE) result in an adverse pregnancy outcome (APO); early identification of women with SLE who are at high risk of APO is vital. We previously derived a risk model for APO using logistic regression and data from the PROMISSE Study, a large multi-center, multiethnic/racial study of APO in women with mild/moderate SLE and/or aPL. While this highly interpretable regression model showed promising predictive performance, we sought to determine if novel and increasingly popular machine learning (ML) approaches would enhance APO risk prediction using all available predictors and potential complex relationships such as interactions or higher order terms. We compared logistic regression modeling to LASSO, a regression approach that handles high-dimensionality and correlated predictors through shrinkage of estimated coefficients, as well as several 'black box' ML algorithms. ML techniques are well-suited to highdimensional data, require no variable selection, and unlike regression-based approaches are able to explore complex relationships without explicit input by the user.

Methods We used the original PROMISSE data (41 predictor variables from 385 subjects) with APO (71/385, 18.4\%) defined as preterm delivery due to placental insufficiency or preeclampsia, fetal or neonatal death, or fetal growth restriction. Logistic regression with stepwise selection (LR-S) was compared to LASSO, random forest (RF), neural network (NN) with 2 hidden neurons, support vector machines with RBF kernel $\left(\mathrm{SVM}_{\mathrm{RBF}}\right)$, and gradient boosting (GB). To summarize discrimination we present the area under the receiver operating curve (AUC), along with sensitivity ( $\mathrm{Sn}$ ) and specificity $(\mathrm{Sp})$ at an optimal cut-point.

Results Regression based classifiers confirmed the predictors of APO identified in our previously reported model: non-white race, use of anti-hypertensive medication, low platelets, SLE disease activity, lupus anticoagulant (LAC) + , and high diastolic blood pressure (DBP). RF additionally revealed two novel interaction variables that increased APO risk: LAC+ with anti- $\beta 2$ GPI IgM, high DBP with low C3. LR-S and LASSO were observed to have similar overall discrimination (AUC $=0.75$ vs. 0.77 , table 1 ) but LASSO had higher sensitivity $(\mathrm{S} n=0.71$ vs. 0.65$)$. ML classifiers $\mathrm{RF}$ and $\mathrm{SVM}_{\mathrm{RBF}}$ had similar good performance $(\mathrm{AUC}=0.77-0.78)$, while $\mathrm{NN}$ and GB were inferior.

Conclusions Several popular ML algorithms did not provide meaningful improvements in the prediction of APO. The strong relative performance of regression-based models with
Abstract 1106 Table 1 Summary of $5 \times 10$ fold cross-validation results

\begin{tabular}{llll}
\hline Model & AUC & Sensitivity & Specificity \\
\hline LR-S & 0.75 & 0.65 & 0.78 \\
LASSO & 0.77 & 0.71 & 0.75 \\
NN & 0.71 & 0.70 & 0.61 \\
$R F$ & 0.77 & 0.77 & 0.78 \\
GB & 0.72 & 0.72 & 0.72 \\
SVM-RBF & 0.78 & 0.78 & 0.73 \\
\hline
\end{tabular}

this large and well-characterized clinical data set is notable as these models are highly interpretable, well-understood, and generally require fewer variables to generate a risk prediction. It is unlikely that complex ML algorithms with existing variables will yield superior APO predictions; new clinical and laboratory markers may improve predictions in the future.

Acknowledgments This work was supported by $\mathrm{NIH}$ grant R21 AR076612

\section{ECONOMIC EVALUATION OF HYDROXYCHLOROQUINE USE IN AN INTERNATIONAL INCEPTION COHORT}

${ }^{1}$ Megan RW Barber, ${ }^{2} Y$ van St Pierre, ${ }^{3} J o h n$ G Hanly, ${ }^{4}$ Murray B Urowitz, ${ }^{5}$ Caroline Gordon, ${ }^{6}$ Sang-Cheol Bae, ${ }^{7}$ Juanita Romero-Diaz, ${ }^{4}$ Jorge Sanchez-Guerrero, ${ }^{8}$ Sasha Bernatsky, ${ }^{9}$ Daniel J Wallace, ${ }^{10}$ David A Isenberg, ${ }^{10}$ Anisur Rahman, ${ }^{11}$ Joan T Merrill, ${ }^{12}$ Paul R Fortin, ${ }^{4}$ Dafna D Gladman, ${ }^{13}$ Ian N Bruce, ${ }^{14}$ Michelle Petri, ${ }^{15}$ Ellen M Ginzler, ${ }^{16}$ Mary Anne Dooley, ${ }^{17}$ Rosalind Ramsey-Goldman, ${ }^{18}$ Susan Manzi, ${ }^{19}$ Andreas Jönsen, ${ }^{20}$ Graciela S Alarcón, ${ }^{21}$ Ronald FVan Vollenhoven, ${ }^{22}$ Cynthia Aranow, ${ }^{22}$ Meggan Mackay, ${ }^{23}$ Guillermo RuizIrastorza, ${ }^{24} \mathrm{~S}$ Sam Lim, ${ }^{25}$ Murat Inanc, ${ }^{26}$ Kenneth C Kalunian, ${ }^{27}$ Soren Jacobsen, ${ }^{28} \mathrm{Christine}$ A Peschken, ${ }^{29}$ Diane L Kamen, ${ }^{30}$ Anca Askanase, ${ }^{1}$ Ann E Clarke*. ${ }^{1}$ University of Calgary, Alberta, Canada; ${ }^{2}$ Research Institute of the McGill University Health Center, Montreal, Canada; ${ }^{3}$ Queen Elizabeth II Health Sciences Centre and Dalhousie University, Halifax, Nova Scotia, Canada; ${ }^{4}$ Centre for Prognosis Studies in the Rheumatic Diseases, Toronto Western Hospital and University of Toronto, Toronto, Ontario, Canada; ${ }^{5}$ Rheumatology Research Group, Institute of Inflammation and Ageing, College of Medical and Dental Sciences, University of Birmingham, Birmingham, UK; ${ }^{6}$ Hanyang University Hospital for Rheumatic Diseases, Seoul, Korea; ${ }^{7}$ Instituto Nacional de Ciencias Médicas y Nutrición, Mexico City, Mexico; ${ }^{8}$ McGill University Health Centre, Montreal, Canada; ${ }^{9}$ Cedars-Sinai/David Geffen School of Medicine at the University of California, Los Angeles, USA; ${ }^{10}$ University College London, London, UK; ${ }^{11}$ Department of Clinical Pharmacology, Oklahoma Medical Research Foundation, OKlahoma City, OK, USA; ${ }^{12} \mathrm{CHU}$ de Québec -Université Laval, Québec City, Canada; ${ }^{13}$ Arthritis Research UK Epidemiology Unit, Institute of Inflammation and Repair, Manchester Academic Health Sciences Centre, the University of Manchester, and NIHR Manchester Musculoskeletal Biomedical Research Unit, Central Manchester University Hospitals National Health Service Foundation Trust, Manchester Academic Health Science Centre, Manchester, UK; ${ }^{14}$ Johns Hopkins University School of Medicine, Baltimore, Maryland, USA; ${ }^{15}$ State University of New York Downstate Medical Center, Brooklyn, New York, USA; ${ }^{16}$ Thurston Arthritis Research Center, University of North Carolina, Chapel Hill, NC, USA; ${ }^{17}$ Northwestern University and Feinberg School of Medicine, Chicago, Illinois, USA; ${ }^{18}$ University of Pittsburgh School of Medicine, Pittsburgh, Pennsylvania, USA; ${ }^{19}$ Lund University, Lund, Sweden; ${ }^{20}$ University of Alabama at Birmingham, USA; ${ }^{21}$ University of Amsterdam, Rheumatology and Immunology Center, Amsterdam, Noord-Holland, NL; ${ }^{22}$ Feinstein Institute for Medical Research, Manhasset, New York, USA; ${ }^{23}$ BioCruces Health Research Institute, Hospital Universitario Cruces, University of the Basque Country, Barakaldo, Spain; ${ }^{24}$ Emory University School of Medicine, Atlanta, Georgia, USA; ${ }^{25}$ Istanbul University, Istanbul, Turkey; ${ }^{26}$ University of California Los Angeles School of Medicine, La Jolla, California, USA; ${ }^{27}$ Copenhagen Lupus and Vasculitis Clinic, Rigshospitalet, Copenhagen University Hospital, Copenhagen, Denmark; ${ }^{28}$ University of Manitoba, Winnipeg, Manitoba, Canada; ${ }^{29}$ Medical University of South Carolina, Charleston, USA; ${ }^{30}$ Hospital for Joint Diseases, New York University Seligman Center for Advanced Therapeutics, New York, New York, USA

10.1136/lupus-2021-lupus21century.50 